TRANSACTIONS OF THE

AMERICAN MATHEMATICAL SOCIETY

Volume 232, 1977

\title{
THE LEVEL STRUCTURE OF A RESIDUAL SET OF CONTINUOUS FUNCTIONS
}

\author{
BY
}

\author{
A. M. BRUCKNER AND K. M. GARG
}

\begin{abstract}
Let $C$ denote the Banach space of continuous real-valued functions on $[0,1]$ with the uniform norm. The present article is devoted to the structure of the sets in which the graphs of a residual set of functions in $C$ intersect with different straight lines.

It is proved that there exists a residual set $A$ in $C$ such that, for every function $f \in A$, the top and the bottom (horizontal) levels of $f$ are singletons, in between these two levels there are countably many levels of $f$ that consist of a nonempty perfect set together with a single isolated point, and the remaining levels of $f$ are all perfect. Moreover, the levels containing an isolated point correspond to a dense set of heights between the minimum and the maximum values assumed by the function.

As for the levels in different directions, there exists a residual set $B$ in $C$ such that, for every function $f \in B$, the structure of the levels of $f$ is the same as above in all but a countable dense set of directions, and in each of the exceptional nonvertical directions the level structure of $f$ is the same but for the fact that one (and only one) of the levels has two isolated points in place of one. For a general function $f \in C$ a theorem is proved establishing the existence of singleton levels of $f$, and of the levels of $f$ that contain isolated points.
\end{abstract}

1. Introduction. In this section, while discussing the results, a few definitions and notations are stated that are used throughout the article.

We denote by $R$ the space of real numbers and by $C$ the space of continuous functions $f:[0,1] \rightarrow R$ equipped with the uniform norm $\|f\|=$ $\sup \{|f(x)|: 0 \leqslant x \leqslant 1\}$. A subset $A$ of $C$ is said to be residual in $C$ if its complement $C \sim A$ is of the first category in $C$.

1.1. Definition. Let $f$ be a given function in $C$ and let $\lambda \in R$. For every $c \in R$, the set $\{x: f(x)=\lambda x+c\}$ is called a level of $f$ in the direction $\lambda$, and when $\lambda=0$ this set is called a horizontal level of $f$. By a level of $f$ we mean, in general, a level of $f$ in some direction $\lambda \in R$.

We further denote by $\alpha_{f, \lambda}$ and $\beta_{f, \lambda}$ the infimum and the supremum, respectively, of the set of real numbers $c$ for which the level $\{x: f(x)=\lambda x+$ c) is nonempty, or, equivalently,

Received by the editors March 25, 1976.

AMS (MOS) subject classifications (1970). Primary 46E15, 26-00; Secondary 26A27, 26 A48.

Key words and phrases. Banach space $C[0,1]$, graph of continuous functions, structure of level sets, perfect levels, derivates, nondifferentiable functions.

- American Mathematical Society 1977 


$$
\alpha_{f, \lambda}=\inf \{f(x)-\lambda x: 0 \leqslant x \leqslant 1\}, \quad \beta_{f, \lambda}=\sup \{f(x)-\lambda x: 0<x<1\} .
$$

It was proved in [4, Theorems $\left.2^{\prime}, 3\right]$ that there exists a residual set $A$ in $C$ such that, for every $f \in A$ and $\lambda \in R$, the level $\{x: f(x)=\lambda x+c\}$ of $f$ in the direction $\lambda$ is uncountable whenever $\alpha_{f, \lambda}<c<\beta_{f, \lambda}$, and that this level is perfect for all but a countable set of values of $c$. It was further proved there [4, Theorem 4] that $C$ contains a dense set of functions of which every horizontal level is perfect, and the question was raised whether such functions form a residual set in $C$.

We first prove in $\$ 2$, strengthening a theorem due to Jarnik [8], that $C$ contains a residual set of functions that are of nonmonotonic type (see Definition 2.1). This yields another version of a theorem of Mazurkiewicz [9] and Banach [1] on the residuality of the set of nondifferentiable functions in C.

Answering the above mentioned question in the negative, it is proved in $\$ 3$ that there exists a residual set of functions in $C$ whose horizontal levels, or more generally the levels in any prescribed direction, are normal in the following sense:

1.2. Definition. The levels of a function $f \in C$ are said to be normal in a direction $\lambda(\in R)$ if there exists a countable dense set $E_{f, \lambda}$ in $\left(\alpha_{f, \lambda}, \beta_{f, \lambda}\right)$ such that the level $\{x: f(x)=\lambda x+c\}$ of $f$ in the direction $\lambda$ is

(i) a perfect set when $c \notin E_{f, \lambda} \cup\left\{\alpha_{f, \lambda}, \beta_{f, \lambda}\right\}$,

(ii) a single point when $c=\alpha_{f, \lambda}$ or $\beta_{f, \lambda}$, and

(iii) the union of a nonempty perfect set $P$ with an isolated point $x \notin P$ when $c \in E_{f, \lambda}$ ( $P$ and $x$ depending on $f, \lambda$ and $c$ ).

In $\S 4$ we study levels in all directions. It is first proved that for every function $f \in C$ there is a countable set $\Lambda$ in $R$ such that for every $\lambda \in R \sim \Lambda$ the level $\{x: f(x)=\lambda x+c\}$ consists of a single point when $c=\alpha_{f, \lambda}$ or $\beta_{f, \lambda}$, and it contains at least one isolated point for a dense set of values of $c$ in $\left(\alpha_{f, \lambda}\right.$, $\left.\beta_{f, \lambda}\right)$. Contrary to some of the assertions of Gillis [6] this shows that there is no continuous function on $[0,1]$ of which all the levels are perfect or infinite.

Finally, we prove that there exists a residual set of functions $f$ in $C$ such that the levels of $f$ are normal in all but a countable dense set of directions $\Lambda_{f}$ in $R$, and in each direction $\lambda \in \Lambda_{f}$ the levels of $f$ are normal except that there is a unique element $c$ of $E_{f, \lambda} \cup\left\{\alpha_{f, \lambda}, \beta_{f, \lambda}\right\}$ for which the level $\{x: f(x)=\lambda x+$ c) contains two isolated points in place of one. The sets of directions $\lambda$ in $\Lambda_{f}$ for which the level $\left\{x: f(x)=\lambda x+\alpha_{f, \lambda}\right\}$ or $\left\{x: f(x)=\lambda x+\beta_{f, \lambda}\right\}$ consists of exactly two points are also dense in $R$.

The space $C$ being complete, this equally proves the existence of continuous functions which have the above unexpected level structure. We have been unable to find any example of such functions in the literature. There are however several known examples of continuous functions that are of non- 
monotonic type, and the levels of such a function are found to be normal in all but countably many directions.

While concluding the introduction, we add a few definitions that are used repeatedly in the sequel.

1.3. Definition. If $f \in C$ and $\lambda \in R$, we denote by $f_{-\lambda}$ [5] the function defined by

$$
f_{-\lambda}(x)=f(x)-\lambda x, \quad 0<x \leqslant 1 .
$$

A function $f$ is said to be of monotonic type [5] if there exists a real number $\lambda$ such that the function $f_{-\lambda}$ is monotone. If $f$ is not of monotonic type in any interval, it is said to be nowhere of monotonic type.

If $f \in C$ and $\varepsilon>0$, the open ball $\{g \in C:\|g-f\|<\varepsilon\}$ of $C$ is denoted as usual by $B(f, \varepsilon)$.

An interval $I \subset[0,1]$ is said to be a rational interval if both of its endpoints are rational, and $I$ will be called an open interval if it is open relative to $[0,1]$.

2. Functions of nonmonotonic type in $C$. In this section we obtain an extension of a theorem of Jarnik [8] that is needed in both the following sections. For this purpose we require the following

2.1. Definition. A function $f \in C$ is said [2] to be nondecreasing at a point $x$ in $[0,1]$ if there exists a $\delta>0$ such that $f(t) \leqslant f(x)$ when $t \in[0$, 1] $\cap(x-\delta, x)$ and $f(t) \geqslant f(x)$ when $t \in[0,1] \cap(x, x+\delta)$. The function $f$ is called nonincreasing at $x$ if $-f$ is nondecreasing at $x$, and $f$ is called monotone at $x$ if it is either nondecreasing or nonincreasing at $x$.

Let $f$ be said to be of monotonic type at $x$ if there exists a real number $\lambda$ such that the function $f_{-\lambda}$ is monotone at $x$. If $f$ is not of monotonic type at any point of its domain, it will be said to be of nonmonotonic type.

If a function $f$ is of monotonic type (see Definition 1.3), it is clearly so at each point of its domain. The converse, however, does not hold in general; consider for example the function $f(x)=\sqrt{x(1-x)}$. The property of $f$ being of nonmonotonic type is, on the other hand, considerably stronger than that of $f$ being nowhere of monotonic type. For there exist continuous functions of bounded variation that are nowhere of monotonic type [3, p. 85], but a function of nonmonotonic type cannot be derivable at any point. In fact, $f$ is not of monotonic type at a point $x$ if and only if it has a "knot point" [5] at $x$, viz. $\underline{D} f(x)=-\infty$ and $\bar{D} f(x)=+\infty$.

2.2. ThEOREM. The functions of nonmonotonic type form a dense $G_{\delta}$ set in $C$.

Proof. Let $A$ denote the set of functions $f$ in $C$ for which there exists a real number $\lambda$ such that the function $f_{-\lambda}$ is nondecreasing at some point of $[0,1]$. Further, if $n$ is any natural number, let $A_{n}$ denote the set of functions $f$ in $C$ 
for which there exist $\lambda \in[-n, n]$ and $x \in[0,1]$ such that $f_{-\lambda}(t) \leqslant f_{-\lambda}(x)$ when $t \in[0,1] \cap(x-1 / n, x)$ and $f_{-\lambda}(t) \geqslant f_{-\lambda}(x)$ when $t \in[0,1] \cap(x$, $x+1 / n)$. It is clear that $A=\cup_{n=1}^{\infty} A_{n}$.

First we show that the set $A_{n}$ is closed and nowhere dense for each $n$. Let $n$ be a given natural number, and let $\left\{f_{k}\right\}$ be any sequence of functions in $A_{n}$ that converges uniformly to some function $f$ in $C$. Then for each $k$ there exist $\lambda_{k} \in[-n, n]$ and $x_{k} \in[0,1]$ such that $f_{-\lambda_{k}}(t) \leqslant f_{-\lambda_{k}}\left(x_{k}\right)$ when $t \in[0$, 1] $\cap\left(x_{k}-1 / n, x_{k}\right)$ and $f_{-\lambda_{k}}(t) \geqslant f_{-\lambda_{k}}\left(x_{k}\right)$ when $t \in[0,1] \cap\left(x_{k}, x_{k}+1 / n\right)$. Clearly, there exists an increasing sequence $\left\{k_{i}\right\}$ of natural numbers such that $\left\{\lambda_{k_{i}}\right\}$ converges to some $\lambda \in[-n, n]$ and $\left\{x_{k_{1}}\right\}$ converges to some $x \in[0,1]$. It can be easily verified that $f_{-\lambda}(t) \leqslant f_{-\lambda}(x)$ when $t \in[0,1] \cap(x-1 / n, x)$ and $f_{-\lambda}(t) \geqslant f_{-\lambda}(x)$ when $t \in[0,1] \cap(x, x+1 / n)$. This proves that $f \in$ $A_{n}$, so that $A_{n}$ is closed in $C$.

To prove that $A_{n}$ is nowhere dense in $C$, it suffices to show that $A_{n}$ does not contain any open subset of $C$. Let $U$ be an arbitrary nonempty open subset of $C$. Then there exists a polynomial $g \in U$ and there exists an $\varepsilon>0$ such that $B(g, \varepsilon) \subset U$. Let $0<\alpha<\varepsilon, \beta=\sup \left\{\left|g^{\prime}(x)\right|: 0 \leqslant x \leqslant 1\right\}$ and let $m$ be an odd integer such that

$$
m>\max \left\{2 n, \frac{3(\beta+n)}{\alpha}\right\} .
$$

Let

$$
h\left(\frac{2 i}{m}\right)=\alpha, \quad h\left(\frac{2 i+1}{m}\right)=0 \quad\left(i=0,1, \ldots, \frac{m-1}{2}\right),
$$

and let $h$ be defined linearly in the interior of each interval $[i / m,(i+1) / m]$, $i=0,1, \ldots, m-1$, so as to be continuous on $[0,1]$. Then the function $f=g+h \in U$. We claim that $f \notin A_{n}$.

Let $x$ be any point of $[0,1]$. There exists an integer $i$ such that $0<i<$ $(m-1) / 2$ and $2 i / m \leqslant x<(2 i+2) / m$. In case $2 i / m \leqslant x<(2 i+1) / m$, we have $x<(2 i+1) / m<x+1 / n$ and

$$
\begin{aligned}
f\left(\frac{2 i+1}{m}\right) & -f(x)=g\left(\frac{2 i+1}{m}\right)-g(x)+h\left(\frac{2 i+1}{m}\right)-h(x) \\
& \leqslant \beta\left(\frac{2 i+1}{m}-x\right)-\frac{\alpha}{1 / m}\left(\frac{2 i+1}{m}-x\right)<-n\left(\frac{2 i+1}{m}-x\right) ;
\end{aligned}
$$

if $(2 i+1) / m<x<(2 i+3 / 2) / m$, we have $x-1 / n<2 i / m<x$ and

$$
\begin{aligned}
& f\left(\frac{2 i}{m}\right)-f(x)=g\left(\frac{2 i}{m}\right)-g(x)+\alpha-h(x) \\
& \quad \geqslant \beta\left(\frac{2 i}{m}-x\right)+\frac{\alpha}{2}>\beta\left(\frac{2 i}{m}-x\right)+\frac{3(\beta+n)}{2 m}>-n\left(\frac{2 i}{m}-x\right)
\end{aligned}
$$


and in case $(2 i+3 / 2) / m \leqslant x<(2 i+2) / m$, we have $x<(2 i+3) / m<x$ $+1 / n$ and

$$
\begin{aligned}
f\left(\frac{2 i+3}{m}\right) & -f(x)=g\left(\frac{2 i+3}{m}\right)-g(x)-h(x) \\
& <\beta\left(\frac{2 i+3}{m}-x\right)-\frac{\alpha}{2}<\beta\left(\frac{2 i+3}{m}-x\right)-\frac{3(\beta+n)}{2 m} \\
< & -n\left(\frac{2 i+3}{m}-x\right) .
\end{aligned}
$$

This proves that $f \notin A_{n}$, and so $A_{n}$ is nowhere dense in $C$.

The set $A$ is thus an $F_{\sigma}$ set of the first category in $C$. If $B$ denotes the set of functions $f$ in $C$ for which there exists a real number $\lambda$ such that the function $f_{-\lambda}$ is nonincreasing at some point of $[0,1]$, we have $B=\{-f: f \in A\}$, so that $B$ is equally an $F_{\sigma}$ set of the first category in $C$. As $C$ is a complete space, it now follows that the set $C \sim(A \cup B)$ of the functions of nonmonotonic type is a dense $G_{\delta}$ set in $C$. Hence the theorem.

Mazurkiewicz [9] and Banach [1] proved in 1931 that $C$ contains a residual set of functions which do not have a finite right or left derivative at any point of $[0,1]$. Here the term "finite" cannot be deleted as it was shown later by Saks [10]. Since a function of nonmonotonic type has a knot point everywhere, Theorem 2.2 yields the following variant of the Mazurkiewicz-Banach theorem:

2.3. Corollary. There exists a residual set of functions in $C$ which do not have a finite or infinite derivative at any point.

Jarnik proved in 1933 (see part 1 of [8, Satz I] and its proof) that the functions $f \in C$ for which at each point of $(0,1)$ every extended real number is a derived number of $f$ form a dense $G_{\delta}$ set in $C$. Combining this with Theorem 2.2 we obtain

2.4. Corollary. The functions $f \in C$ for which at each point of $[0,1]$ every extended real number is a derived number of $f$ form a dense $G_{\delta}$ set in $C$.

3. Levels in one direction. The following definitions are standard, but are recalled here for the sake of definiteness.

3.1. Definition. Given a function $f \in C$, a point $x \in[0,1]$ is said to be a point of (relative) maximum of $f$ if there exists a $\delta>0$ such that $f(t) \leqslant f(x)$ whenever $t \in[0,1] \cap(x-\delta, x+\delta)$, and $x$ is a point of proper maximum of $f$ if there exists a $\delta>0$ such that $f(t)<f(x)$ whenever $t \in[0,1] \cap(x-\delta$, $x+\delta) \sim\{x\}$. If $x$ is a point of maximum or proper maximum of $-f$, then $f$ is said to have a minimum or a proper minimum at $x$ respectively. Further, $x$ is said to be a point of extremum of $f$ if $f$ has a maximum or a minimum at $x$, 
and $x$ is a point of proper extremum of $f$ if $f$ has a proper maximum or a proper minimum at $x$.

3.2. LemMA. There exists a residual set of functions $f$ in $C$ such that no horizontal level of $f$ contains more than one point of extremum of $f$.

Proof. If $I$ and $J$ are any two disjoint closed rational subintervals of $[0,1]$, let $A_{I, J}$ denote the set of functions $f$ in $C$ for which neither the supremum nor the infimum of $f$ on $I$ is equal to either the supremum or the infimum of $f$ on $J$. Let, further, $A=\cap A_{I, J}$, where the intersection is taken over all pairs of disjoint closed rational subintervals of $[0,1]$. As this intersection is countable, and the functions in $A$ clearly have the required property, it suffices to show that the set $A_{I, J}$ is residual in $C$ for each pair $(I, J)$.

Let $(I, J)$ be a given pair of disjoint closed rational subintervals of $[0,1]$. Let $E_{1}$ denote the set of functions $f$ in $C$ for which

$$
\sup \{f(x): x \in I\} \neq \sup \{f(x): x \in J\}
$$

and let $E_{2}, E_{3}$ and $E_{4}$ denote the other three sets obtained by replacing the supremum by infimum on $I$ or $J$, or both. Clearly, $A_{I, J}=\cap_{i=1}^{4} E_{i}$.

Let $f \in E_{1}, \alpha=\sup \{f(x): x \in I\}$ and $\beta=\sup \{f(x): x \in J\}$. Then $\alpha \neq$ $\beta$. Putting $\varepsilon=|\alpha-\beta|$, it may be easily seen that the open ball $B(f, \varepsilon / 2)$ is contained in $E_{1}$. This proves that the set $E_{1}$ is open in $C$. As $E_{1}$ is clearly dense in $C$, it follows that $E_{1}$ is residual in $C$. The sets $E_{i}(i=2,3,4)$ are proved similarly to be residual in $C$, so that the set $A_{I, J}$ is residual in $C$. This completes the proof of the lemma.

3.3. THEOREM. There exists a residual set of functions in $C$ whose horizontal levels are normal.

Proof. Let $A$ denote the intersection of the two residual sets in $C$ determined by Theorem 2.2 and Lemma 3.2. We claim that every function in the residual set $A$ has normal horizontal levels.

Let $f \in A$, and let

$$
\alpha=\alpha_{f, 0} \equiv \inf \{f(x): 0<x \leqslant 1\}, \quad \beta=\beta_{f, 0} \equiv \sup \{f(x): 0 \leqslant x \leqslant 1\} .
$$

By Theorem 2.2, the function $f$ is not monotone at any point of $[0,1]$. Hence, for every $c \in R$, a point $x \in f^{-1}(c)$ is an isolated point of the level $f^{-1}(c)$ if and only if $x$ is a point of proper extremum of $f$. Also, by Lemma 3.2, each horizontal level of $f$ contains at most one point of extremum of $f$. Consequently, every point of extremum of $f$ is a point of proper extremum of $f$.

As the function $f$ is continuous and nowhere monotone, it has a dense set of extrema $D$ in $[0,1]$. Since every point of $D$ is a proper extremum of $f, D$ is countable, and its image $f(D)$ is a countable dense subset of $[\alpha, \beta]$. Also, since $f$ attains $\alpha$ and $\beta$, we have $\alpha, \beta \in f(D)$. Let $E=f(D) \sim\{\alpha, \beta\}$. 
Let $c$ be any real number. When $c \notin E \cup\{\alpha, \beta\}=f(D)$, the level $f^{-1}(c)$ does not contain any point of extremum of $f$, and so $f^{-1}(c)$ has no isolated point, i.e. $f^{-1}(c)$ is perfect. Since no horizontal level of $f$ contains more than one point of extremum of $f$, the levels $f^{-1}(\alpha)$ and $f^{-1}(\beta)$ are clearly singleton sets. When $c \in E$, the level $f^{-1}(\alpha)$ contains one and only one point of extremum of $f$, say $x$. Then $x$ is clearly an isolated point of $f^{-1}(c)$. Further, due to the Darboux property of $f$, the level $f^{-1}(c)$ contains points other than $x$, each of which must be a limit point of $f^{-1}(c)$. Hence $f^{-1}(c) \sim\{x\}$ is a nonempty perfect set. This proves that the horizontal levels of $f$ are normal, and the proof of the theorem is complete.

3.4. Corollary. Given a function $\phi \in C$, there exists a residual set $A$ in $C$ such that, for every function $f \in A$, the horizontal levels of the function $f-\phi$ are normal.

Proof. There exists, by the above theorem, a residual set $B$ in $C$ such that the horizontal levels of each function $f \in B$ are normal. As every residual set contains a dense $G_{\delta}$ set, there further exists a sequence of dense open sets $\left\{U_{n}\right\}$ in $C$ such that $\cap_{n=1}^{\infty} U_{n} \subset B$. Clearly, for each $n$, the set $\phi+U_{n}$ is again dense and open in $C$, and so the set $A=\cap_{n=1}^{\infty}\left(\phi+U_{n}\right)$ is residual in $C$. If $f \in A$, then $f-\phi \in U_{n}$ for each $n$, so that $f-\phi \in B$.

3.5. CoRollary. If a function $\phi \in C$ is not zero at any point of $[0,1]$, then there exists a residual set $A$ in $C$ such that, for every function $f \in A$, the horizontal levels of the function $f / \phi$ are normal.

Proof. Let $B$ and $\left\{U_{n}\right\}$ be as defined above, and let this time $A=$ $\bigcap_{n=1}^{\infty}\left(\phi U_{n}\right)$, where $\phi U$ denotes the set $\{\phi f: f \in U\}$ whenever $U \subset C$. If $f \in A$, we have $f / \phi \in \cap_{n=1}^{\infty} U_{n} \subset B$, and so the horizontal levels of $f / \phi$ are normal. To prove that $A$ is residual in $C$, it suffices to show that $\phi U$ is dense and open in $C$ whenever $U$ is so.

Let $U$ be any dense open set in $C$, and let $\alpha=\inf \{|\phi(x)|: 0<x \leqslant 1\}$. Given $f \in \phi U$, we have $f / \phi \in U$, and so there exists an $\varepsilon>0$ such that $B(f / \phi, \varepsilon) \subset U$. For every function $g \in B(f, \alpha \varepsilon)$ we have

$$
\left\|\frac{g}{\phi}-\frac{f}{\phi}\right\| \leqslant \frac{\|g-f\|}{\alpha}<\varepsilon,
$$

so that $g / \phi \in U$, or $g \in \phi U$. This proves that $\phi U$ is open. If $V$ is any given nonempty open set in $C$, it follows by the same argument that $(1 / \phi) V$ is open in $C$, and so, $U$ being dense in $C$, we have $U \cap\{(1 / \phi) V\} \neq \varnothing$. From this it follows that $(\phi U) \cap V \neq \varnothing$, which proves that $\phi U$ is dense in $C$.

3.6. REMARK. Given $\lambda \in R$, on putting $\phi(x)=\lambda x(0<x<1)$ in Corollary 3.4 it follows that there is a residual set of functions in $C$ whose levels are 
normal in the direction $\lambda$. Consequently, for every sequence of real numbers $\left\{\lambda_{n}\right\}$, there exists a residual set of functions in $C$ whose levels are normal in each of the directions $\lambda_{n}(n=1,2, \ldots)$. This result is not comparable with Theorem 4.8 of the following section, where a residual set of functions in $C$ is proved to have its levels normal in all but a countable set of directions; for the set of exceptional directions varies there with the function in the residual set.

Let $a$ and $b$ be two real numbers such that either (i) $a b>0$, or (ii) $a b<0$ and $|a|<|b|$. Let further, for every function $f \in C, \alpha_{f}$ and $\beta_{f}$ denote the infimum and the supremum respectively of the set of real numbers $c$ for which the level $\{x: f(x)=c a x+c b\}$ of $f$ is nonempty. On putting $\phi(x)=a x$ $+b(0<x<1)$ in Corollary 3.5, it follows that there exists a residual set of functions $f$ in $C$ for which there is a countable dense set $E_{f}$ in $\left(\alpha_{f}, \beta_{f}\right)$ such that the level $\{x: f(x)=c a x+c b\}$ of $f$ is (i) a perfect set when $c \notin E_{f} \cup$ $\left\{\alpha_{f}, \beta_{f}\right\}$, (ii) a single point when $c=\alpha_{f}$ or $\beta_{f}$, and (iii) the union of a nonempty perfect set with a point outside that set when $c \in E_{f}$.

\section{Levels in different directions.}

4.1. Definition. Let $f \in C, I$ be a subinterval of $[0,1], \Gamma$ be the graph of the restriction of $f$ to $I$, and let $L$ be a line $y=\lambda x+c$. The line $L$ is said to support the graph of $f$ in $I$ from above [below] if $f$ is a supporting line (i.e. a supporting hyperplane) of $\Gamma$ from above [below], viz. if $f(x)<\lambda x+c$ [ $>\lambda x$ $+c$ ] for every $x \in I$ and there exists a point $x_{0}$ in $I$ such that $f\left(x_{0}\right)=\lambda x_{0}+$ $c$. Further, if the point $x_{0}$ is not unique, then $L$ is said to support the graph of $f$ in $I$ from above [below] at more than one point. By saying that $L$ supports the graph of $f$ in $I$ it is meant that $L$ does so either from above or from below.

If $I$ and $J$ are two disjoint subintervals of [0,1], a line will be said to support the graph of $f$ in $I$ and $J$ if its supports the graph of $f$ in $I$ as well as the graph of $f$ in $J$. Similarly for three or more mutually disjoint subintervals of $[0,1]$.

It may be recalled here that we call a subinterval of $[0,1]$ open if it is open relative to $[0,1]$.

4.2. LEMMA. For every function $f \in C$ there are at most countably many lines that support the graph of $f$ in two (or more) disjoint open subintervals of $[0,1]$.

Proof. Let $\mathcal{L}$ denote the set of lines that support the graph of $f$ in at least two disjoint open subintervals of $[0,1]$. For every line $L \in \mathcal{L}$ there exist two disjoint open rational subintervals $I$ and $J$ of $[0,1]$ such that $L$ supports the graph of $f$ in $I$ and $J$. It is easy to see that there is at most one line that supports the graph of $f$ in $I$ and $J$ from above (or below), and there is at most one line that supports the graph of $f$ in $I$ from above [below] and in $J$ from below [above]. Thus there are at most four lines that support the graph of $f$ in 
$I$ and $J$. As the set of pairs of rational subintervals of $[0,1]$ is countable, it follows that $\mathcal{L}$ is countable.

From the above lemma we obtain the following result on the existence of isolated points in the levels of a general continuous function.

4.3. THEOREM. For every function $f \in C$ there exists a countable set $\Lambda$ in $R$ such that, for every $\lambda \in R \sim \Lambda$,

(a) the levels $\left\{x: f(x)=\lambda x+\alpha_{f, \lambda}\right\}$ and $\left\{x: f(x)=\lambda x+\beta_{f, \lambda}\right\}$ consist of single points and

(b) there exists a dense set of points $c$ in $\left(\alpha_{f, \lambda}, \beta_{f, \lambda}\right)$ such that the level $\{x$ : $f(x)=\lambda x+c\}$ contains at least one isolated point.

Furthermore, the part (b) is valid for every continuous function $f$ defined on any subinterval of $R$.

Proof. Let $f \in C$, and let $\Lambda$ be the set of slopes of the lines that support the graph of $f$ in at least two disjoint open subintervals of $[0,1]$. Then $\Lambda$ is countable by the above lemma. Let $\lambda \in R \sim \Lambda$.

For every $c \in R$ we have

$$
\{x: f(x)=\lambda x+c\}=\left\{x: f_{-\lambda}(x)=c\right\},
$$

and if $x_{0}$ is a point of this level, it is clear that the line $y=\lambda x+c$ supports the graph of $f$ in some neighborhood of $x_{0}$ if and only if the function $f_{-\lambda}$ has an extremum at $x_{0}$. Hence there is no horizontal level of $f_{-\lambda}$ which contains more than one point of extremum of the function, and consequently $f_{-\lambda}$ has only proper extrema. As the function $f_{-\lambda}$ attains its infimum and supremum in $[0,1]$, the part (a) is now obvious.

Let $(a, b)$ be any open subinterval of $\left(\alpha_{f, \lambda}, \beta_{f, \lambda}\right)$, and let $I$ be a connected component of the open set $\left\{x: a<f_{-\lambda}(x)<b\right\}$. Then $I$ is an open subinterval of $[0,1]$, and $f_{-\lambda}$ is clearly not constant in any subinterval of $I$. Hence if $f_{-\lambda}$ is monotone in $I$, for every $c \in f_{-\lambda}(I)$ we have $a<c<b$ and the point of $I$ where $f_{-\lambda}$ assumes $c$ is an isolated point of the level (*). In case $f_{-\lambda}$ is not monotone in $I$, it has a proper extremum at some point $x_{0}$ in $I$, and putting $c=f_{-\lambda}\left(x_{0}\right)$ we have, once again, $a<c<b$ and this time $x_{0}$ is an isolated point of (*). This proves the part (b).

The above proofs of Lemma 4.2 and part (b) are found to remain valid for every continuous function $f$ defined on any subinterval of $R$.

4.4. REMARK. Gillis constructed a continuous real-valued periodic function $f$ on $R$ for which he claimed [6, Remarks (b), (c)] that all of the levels are perfect, and that the levels of the restriction of $f$ to $[0,1]$ are in turn all infinite. As it is clear from the two parts of Theorem 4.3, both these claims are false. It is however true that all the levels of $f$ in itself are infinite, but this holds for much simpler functions like $f(x)=x^{2} \sin 1 / x$. Using Gillis' first 
assertion it was proved in [4, Theorem 5] that $C$ contains a dense set of functions for which in each direction all but a finite number of levels are perfect. This is again false by Theorem 4.3, part (b).

We need a few more lemmas to prove the next theorem.

4.5. Lemma. There exists a residual set of functions $f$ in $C$ such that, for every open rational interval $I \subset[0,1]$, the slopes of the lines that support the graph of $f$ in I from above at more than one point form a dense set in $R$, and the same holds for the lines that support the graph of $f$ in I from below at more than one point.

Proof. As the set of rational subintervals of $[0,1]$ is countable, it suffices to prove the result for one fixed open rational interval $I \subset[0,1]$.

For every natural number $n$, let $A_{n}$ denote the set of functions $f$ in $C$ for which there exists a line $L$ with slope in $(-1 / n, 1 / n)$ such that $L$ supports the graph of $f$ in $I$ from above at more than one point. We first prove that the set $A_{n}$ is residual in $C$ for each $n$.

Let $n$ be a given natural number, $U$ be any nonempty open subset of $C$, and let $f \in U$. There exists an $\varepsilon>0$ such that $B(f, \varepsilon) \subset U$. Let $\alpha=$ $\sup \{f(x): x \in I\}$. There exists a nondegenerate subinterval $J$ of $I$ such that $f(x)>\alpha-\varepsilon / 4$ for every $x \in J$. Let $\delta$ be a positive number such that the length of $J$ is $>4 \delta$, and let $a$ and $b$ denote the left and right endpoints of $I$ respectively. Then we can find five points $x_{i}(i=0,1, \ldots, 4)$ in $J$ such that $a<x_{0}<x_{1}<x_{2}<x_{3}<x_{4}<b$ and $x_{i}-x_{i-1}=\delta$ for $i=1, \ldots, 4$. Let

$$
\begin{aligned}
& g(x)=f(x) \quad \text { when } x \in\left[0, x_{0}\right] \cup\left[x_{4}, 1\right], \\
& g\left(x_{1}\right)=g\left(x_{3}\right)=\alpha+\varepsilon / 2, \quad g\left(x_{2}\right)=\alpha,
\end{aligned}
$$

and let $g$ be defined linearly in the interior of each interval $\left[x_{i-1}, x_{i}\right]$, $i=1, \ldots, 4$, so as to be continuous on $[0,1]$. Let, further,

$$
\eta=\min \left\{\frac{\delta}{n}, \frac{\delta \varepsilon}{4\left(x_{2}-a\right)}, \frac{\delta \varepsilon}{4\left(b-x_{2}\right)}\right\} .
$$

Since $\left(x_{2}-a\right)>\left(x_{2}-x_{0}\right)=2 \delta$, we have $\eta<\varepsilon / 8$, and so

$$
B(g, \eta) \subset B(f, \varepsilon) \subset U .
$$

We claim that $B(g, \eta) \subset A_{n}$.

Let $h \in B(g, \eta)$, and let $K$ be the convex hull of the graph of $h$ in $\left[x_{0}, x_{4}\right]$. Clearly, $K$ is compact. Let $P$ be the highest point of $K$ with $x$-coordinate $x_{2}$, and let $\beta$ denote the $y$-coordinate of $P$. For each of $i=1$ and 3 we have

$$
h\left(x_{i}\right)>g\left(x_{i}\right)-\eta=\alpha+\varepsilon / 2-\eta,
$$

and so $\beta>\alpha+\varepsilon / 2-\eta$. Also, $h\left(x_{2}\right)<g\left(x_{2}\right)+\eta=\alpha+\eta$, so that

$$
\beta-h\left(x_{2}\right)>\varepsilon / 2-2 \eta>\varepsilon / 2-\varepsilon / 4>0 \text {. }
$$


Thus $P$ is not on the graph of $h$, and so it is a convex sum of two points $S$ and $T$ on the graph of $h$ in $\left[x_{0}, x_{4}\right]$. Let $L$ be the line that contains $S$ and $T$. It is clear that $L$ supports the graph of $h$ in $\left[x_{0}, x_{4}\right]$ from above at more than one point. Since

$$
\alpha+\varepsilon / 2-\eta<h\left(x_{i}\right)<\alpha+\varepsilon / 2+\eta \text { for } i=1,3,
$$

it is easy to see that the slope of $L$ belongs to the interval $(-\eta / \delta, \eta / \delta)$, which is only a subinterval of $(-1 / n, 1 / n)$.

It remains to show that $L$ supports the graph of $h$ in the entire interval $I$. If $x \in\left[a, x_{0}\right]$ we have

$$
h(x)<g(x)+\eta=f(x)+\eta \leqslant \alpha+\eta,
$$

and the slope of the line joining the point $(a, \alpha+\eta)$ with $P$ is

$$
\begin{aligned}
\frac{\beta-(\alpha+\eta)}{x_{2}-a} & >\frac{(\alpha+\varepsilon / 2-\eta)-(\alpha+\eta)}{x_{2}-a}=\frac{\varepsilon-4 \eta}{2\left(x_{2}-a\right)} \\
& >\frac{\varepsilon-\varepsilon / 2}{2\left(x_{2}-a\right)}=\frac{\varepsilon}{4\left(x_{2}-a\right)}>\frac{\eta}{\delta},
\end{aligned}
$$

which in turn is greater than the slope of $L$. Hence $L$ does not meet the graph of $h$ in $\left[a, x_{0}\right]$. A similar argument shows that $L$ does not meet the graph of $h$ in $\left[x_{4}, b\right]$ as well, and so $L$ does support the graph of $h$ in $I$ from above. This proves that $h \in A_{n}$, i.e. $B(g, \eta) \subset A_{n}$. Thus $A_{n}$ contains a dense open subset of $C$, and so it is residual in $C$.

Now, let $\left\{\lambda_{n}\right\}$ be an enumeration of the set of all rational numbers. For every natural number $n$, let $B_{n}$ denote the set of functions $f$ in $C$ for which $f_{-\lambda_{n}} \in A_{n}$. Then $B_{n}$ is again residual in $C$ for each $n$, and so, in turn, is the set $B=\cap_{n=1}^{\infty} B_{n}$.

Let $f \in B$ and $(p, q)$ be a given subinterval of $R$. Let, further, $r=$ $(p+q) / 2$ and $s=(q-p) / 4$. As the interval $(r-s, r+s)$ contains infinitely many points of the sequence $\left\{\lambda_{n}\right\}$, we can find a natural number $n>1 / s$ such that $\lambda_{n} \in(r-s, r+s)$. Since $f \in B_{n}$, the function $f_{-\lambda_{n}}$ is in $A_{n}$, and so there exists a line $L$ with slope in $(-1 / n, 1 / n)$ such that $L$ supports the graph of $f_{-\lambda_{n}}$ in $I$ from above at more than one point. Adding $\lambda_{n}$ to the slope of $L$ we obtain another line $M$ such that its slope $\lambda \in \lambda_{n}-1 / n$, $\left.\lambda_{n}+1 / n\right)$ and such that $M$ supports the graph of $f$ in $I$ from above at more than one point. Clearly,

$$
|\lambda-r| \leqslant\left|\lambda-\lambda_{n}\right|+\left|\lambda_{n}-r\right|<1 / n+s<2 s,
$$

so that $\lambda \in(p, q)$. This proves that the functions in $B$ possess the required property regarding lines that support their graphs from above.

The set $A=B \cap\{-f: f \in B\}$ is again residual in $C$, and each function in $A$ possesses the other required property as well. 
4.6. LemMA. There exists a residual set of functions $f$ in $C$ for which there is no line that supports the graph of $f$ in more than two mutually disjoint open subintervals of $[0,1]$.

Proof. Let $A$ denote the set of functions in $C$ which do not have the required property. If $I, J$ and $K$ are three mutually disjoint open rational subintervals of $[0,1]$, let $A_{I, J, K}$ denote the set of functions $f$ in $C$ for which there exists a line $L$ that supports the graph of $f$ in $I, J$ and $K$. Then $A=\cup A_{I, J, K}$, where the union is taken over all triples $(I, J, K)$ of mutually disjoint open rational subintervals of $[0,1]$. Given such a triple $(I, J, K)$, let further $E$ denote the set of functions $f \in A_{I, J, K}$ for which the corresponding line $L$ supports the graph of $f$ in $I, J$ and $K$ from above. We first show that $E$ is nowhere dense in $C$.

Let $U$ be a given open subset of $C$, and suppose that there exists a function $f$ in $U$ such that $f \in E$. Then there exists a line $y=\lambda x+c$ that supports the graph of $f$ in $I, J$ and $K$ from above, and there exists an $\varepsilon>0$ such that $B(f, \varepsilon) \subset U$. We may assume, without loss of generality, that the interval $J$ is between $I$ and $K$. Let $g$ be a function in $B(f, 3 \varepsilon / 4)$ such that $g$ agrees with $f$ in the intervals $I$ and $K$ and $g=f-\varepsilon / 2$ in $J$. Let $h \in B(g, \varepsilon / 4)$, and suppose that there is a line $L$ that supports the graph of $h$ in $I$ and $K$ from above. It is easy to see that, relative to the interval $J$, the line $L$ is strictly above the line $y=\lambda x+c-\varepsilon / 4$, whereas the graph of $h$ is below this line. Thus $L$ cannot support the graph of $h$ in $J$, i.e. $h \notin E$, or $E \cap$ $B(g, \varepsilon / 4)=\varnothing$. Since $B(g, \varepsilon / 4) \subset B(f, \varepsilon) \subset U$, this proves that $E$ is nowhere dense in $C$.

The other seven subsets of $A_{I, J, K}$ obtained by replacing the support from above in the definition of $E$ by support from below in some or all of the intervals $I, J$ and $K$ are proved similarly to be nowhere dense in $C$. The set $A_{I, J, K}$ is therefore nowhere dense in $C$, and from this it follows that $A$ is of the first category in $C$.

4.7. LEMMA. There exists a residual set of functions $f$ in $C$ for which there are no two distinct parallel lines such that each of these lines supports the graph of $f$ in two disjoint open subintervals of $[0,1]$.

Proof. Let $A$ denote the set of functions in $C$ which do not have the required property. If $I, J, K$ and $L$ are four mutually disjoint open rational subintervals of $[0,1]$, let $A_{I, J, K, L}$ denote the set of functions $f$ in $C$ for which there exist two distinct parallel lines $M$ and $N$ such that $M$ supports the graph of $f$ in $I$ and $J$ and $N$ supports the graph of $f$ in $K$ and $L$. Then we have $A=\cup A_{I, J, K, L}$, where the union is taken over all ordered quadruples $(I, J, K$, $L$ ) of mutually disjoint open rational subintervals of $[0,1]$. Given such a quadruple $(I, J, K, L)$, let further $E$ denote the set of functions $f \in A_{I, J, K, L}$ 
for which the corresponding lines $M$ and $N$ support the graph of $f$ in $I, J$ and $K, L$ respectively from above. We show that $E$ is nowhere dense in $C$.

Let $U$ be a given open subset of $C$, and suppose that there is a function $f$ in $U$ such that $f \in E$. Then there exist two distinct parallel lines $M$ and $N$ such that $M$ supports the graph of $f$ in $I$ and $J$ from above and $N$ supports the graph of $f$ in $K$ and $L$ from above. We may assume, without loss of generality, that the interval $I$ is on the left of $J$ and that $K$ is on the left of $L$. There further exists an $\varepsilon>0$ such that $B(f, \varepsilon) \subset U$. Let $g$ be a function in $B(f, 3 \varepsilon / 4)$ such that $g=f+\varepsilon / 2$ in the intervals $I$ and $L$ and $g=f-\varepsilon / 2$ in $J$ and $K$.

Let $h \in B(g, \varepsilon / 4)$, and suppose that there are two lines $M^{\prime}$ and $N^{\prime}$ such that $M^{\prime}$ supports the graph of $h$ in $I$ and $J$ from above and $N^{\prime}$ supports the graph of $h$ in $K$ and $L$ from above. As the graph of $h$ in $I$ contains points above $M$ and the graph of $h$ in $J$ is entirely below $M$, the slope of $M^{\prime}$ is clearly less than that of $M$. Similarly, the slope of $N^{\prime}$ is greater than that of $N$. Since $M$ and $N$ are parallel, it follows that $M^{\prime}$ and $N^{\prime}$ are not parallel, whence $h \notin E$. As $B(g, \varepsilon / 4) \subset B(f, \varepsilon) \subset U$, this proves that $E$ is nowhere dense in $C$.

The other fifteen subsets of $A_{I, J, K, L}$ obtained by replacing the support from above in the definition of $E$ by support from below in some or all of the intervals $I, J, K$ and $L$ are proved similarly to be nowhere dense in $C$. The set $A_{I, J, K, L}$ is therefore nowhere dense in $C$, and from this it follows that $A$ is of the first category in $C$.

4.8. THEOREM. There exists a residual set of functions $f$ in $C$ for which there is a countable dense set $\Lambda_{f}$ in $R$ such that

(i) the levels of $f$ are normal in each direction $\lambda \in R \sim \Lambda_{f}$ and

(ii) in any direction $\lambda \in \Lambda_{f}$, the levels of $f$ are normal but for the fact that there is a unique element $c_{f, \lambda}$ of $E_{f, \lambda} \cup\left\{\alpha_{f, \lambda}, \beta_{f, \lambda}\right\}$ for which the level $\{x$ : $\left.f(x)=\lambda x+c_{f, \lambda}\right\}$ contains two isolated points in place of one.

Proof. Let $A$ be the intersection of the four residual sets in $C$ determined by Theorem 2.2 and Lemmas $4.5,4.6$ and 4.7. Then $A$ is again residual in $C$, and we shall show that each function in $A$ has the required properties.

Let $f \in A$, and let $\Lambda$ denote the set of slopes of the lines that support the graph of $f$ in at least two disjoint open subintervals of $[0,1]$. Then according to Lemmas 4.2 and 4.5, $\Lambda$ is a countable dense subset of $R$.

Let $\lambda$ be any real number. For every $c \in R$ we have

$$
\{x: f(x)=\lambda x+c\}=\left\{x: f_{-\lambda}(x)=c\right\} .
$$

Hence a subset $E$ of $[0,1]$ is a level of $f$ in the direction $\lambda$ if and only if $E$ is a horizontal level of the function $f_{-\lambda}$. According to Theorem 2.2, the function $f_{-\lambda}$ is not monotone at any point of $[0,1]$. Consequently, a point $x$ in a 
horizontal level of $f_{-\lambda}$ is an isolated point of the level if and only if $f_{-\lambda}$ has a proper extremum at $x$. It is further clear that a point $x_{0}$ in the level $\{x$ : $\left.f_{-\lambda}(x)=c\right\}$ is a point of extremum of $f_{-\lambda}$ if and only if the line $y=\lambda x+c$ supports the graph of $f$ in some neighborhood of $x_{0}$.

When $\lambda \in R \sim \Lambda$, there is no line with slope $\lambda$ that supports the graph of $f$ in two disjoint open subintervals of $[0,1]$. Hence there is no horizontal level of $f_{-\lambda}$ which contains more than one point of extremum of the function. Since $f_{-\lambda}$ is a continuous nowhere monotone function with only proper extrema, it follows by the argument used earlier for Theorem 3.3 that the horizontal levels of $f_{-\lambda}$ are normal. Consequently, the levels of $f$ are normal in the direction $\lambda$.

Now let $\lambda \in \Lambda$. According to Lemma 4.6 there is no line that supports the graph of $f$ in more than two mutually disjoint open subintervals of $[0,1]$. Hence there is no horizontal level of $f_{-\lambda}$ which contains more than two points of extrema of the function. This in turn implies that $f_{-\lambda}$ has only proper extrema. Since $\lambda$ is an element of $\Lambda$, there exists a real number $c_{0}$ such that the line $y=\lambda x+c_{0}$ supports the graph of $f$ in at least two disjoint open subintervals of $[0,1]$. Hence the level $\left\{x: f_{-\lambda}(x)=c_{0}\right\}$ contains, by the above, exactly two points of extrema of $f_{-\lambda}$. For a value of $c$ other than $c_{0}$, the level $\left\{x: f_{-\lambda}(x)=c\right\}$ contains, by Lemma 4.7, at most one point of extremum of $f_{-\lambda}$. The rest follows once again from the fact that $f_{-\lambda}$ is a continuous nowhere monotone function with only proper extrema.

4.9. REMARK. Let $f$ be any function in the above residual subset $A$ of $C$, and let $\lambda \in \Lambda_{f}$. It is clear from the above proof that when $c_{f, \lambda}=\alpha_{f, \lambda}$ or $\beta_{f \lambda}$, the level $\left\{x: f(x)=\lambda x+c_{f, \lambda}\right\}$ of $f$ consists of exactly two points, and when $c_{f, \lambda} \in E_{f, \lambda}$ this level is the union of a nonempty perfect set with two points outside that set. Moreover, if $\Lambda_{f, 1}, \Lambda_{f, 2}$ and $\Lambda_{f, 3}$ denote the sets of values of $\lambda$ in $\Lambda_{f}$ for which $c_{f, \lambda}=\alpha_{f, \lambda}, c_{f, \lambda}=\beta_{f, \lambda}$ or $c_{f, \lambda} \in E_{f, \lambda}$ holds respectively, it follows from Lemma 4.5 that each of the sets $\Lambda_{f, 1}, \Lambda_{f, 2}$ and $\Lambda_{f, 3}$ is dense in $R$. Theorem 4.8 also yields corollaries similar to those of Theorem 3.3.

As for the local behaviour of a residual set of functions in $C$, more can be said besides Theorem 2.2. A function $f \in C$ is said [2] to be "oscillating" on the left [right] of a point $x \in[0,1]$ if for every $\delta>0$ there exist two points $x_{1}$ and $x_{2}$ in $(x-\delta, x)[(x, x+\delta)]$ such that $f\left(x_{1}\right)<f(x)<f\left(x_{2}\right)$. If $f$ is any function in the above residual subset $A$ of $C$, then for every $\lambda \in R$ the function $f_{-\lambda}$ is oscillating, on one side at least, at all but a countable set of points in $[0,1]$. For, as we saw in the proof of Theorem 4.8 , the function $f_{-\lambda}$ is not monotone at any point and it has only proper extrema, which in turn are countable. This may be compared with the local properties of general continuous nowhere monotone functions obtained in Theorem 1 of [2] and Theorem 5 of [3]. 
Employing the argument used in the first half of the proof of Theorem 4.8, we further obtain from Lemma 4.2,

4.10. THEOREM. If a function $f \in C$ is of nonmonotonic type, then its levels are normal in all but a countable set of directions.

4.11. Remark. Many of the known examples of continuous periodic nondifferentiable functions on $R$, including the Weierstrass function, are indeed of nonmonotonic type (see Hobson [7, pp. 403-404 and 407-412]). Although such a function $f$ need not be of nonmonotonic type relative to $[0,1]$, there exists a residual set of points in $R$ where we have $D_{-} f=D_{+} f=-\infty$ and $D^{-} f=D^{+} f=+\infty$ (see [3, Theorem 5]). Choosing two such points $a, b$, $a<b$, it is clear that $f$ is of nonmonotonic type relative to $[a, b]$, and so $f(a+\overline{b-a} x), 0 \leqslant x \leqslant 1$, is a function of nonmonotonic type in $C$.

\section{REFERENCES} 174-179.

1. S. Banach, Über die Bairesche Kategorie gewisser Funktionenmengen, Studia Math. 3 (1931),

2. K. M. Garg, On nowhere monotone functions. I. Derivates at a residual set, Ann. Univ. Sci. Budapest, Eötvös Sect. Math. 5 (1962), 173-177. MR 26 \#3839.

3. On nowhere monotone functions. III. Functions of first and second species, Rev. Math. Pures Appl. 8 (1963), 83-90. MR 27 \# 1545.

4. _ On a residual set of continuous functions, Czechoslovak Math. J. 20 (95) (1970), 537-543. MR 42 \#3233.

5. On bilateral derivates and the derivative, Trans. Amer. Math. Soc. 210 (1975), 295-329. MR 51 \#5861.

6. J. Gillis, Note on a conjecture of Erdös, Quart. J. Math. Oxford Ser. 10 (1939), 151-154.

7. E. W. Hobson, The theory of functions of a real variable and the theory of Fourier's series, Vol. II, Dover, New York, 1958. MR 19 \# 1166.

8. V. Jarnik, Über die Differenzierbarkeit stetiger Funktionen, Fund. Math. 21 (1933), 48-58.

9. S. Mazurkiewicz, Sur les fonctions non dérivables, Studia Math. 3 (1931), 91-94.

10. S. Saks, On the functions of Besicovitch in the space of continuous functions, Fund. Math. 19 (1932), 211-219.

\footnotetext{
Department of Mathematics, University of California, Santa barbara, California 93106
}

Department of Mathematics, University of Alberta, Edmonton, Alberta t6G 2G1, CANADA 